\title{
BOTANICAL RESULTS OF THE ARCHBOLD EXPEDITIONS NEW GUINEA RECORDS OF ANNONACEAE AND MENISPERMACEAE
}

\section{Diels}

\section{MENISPERMACEAE}

Tinospora polygonoides Diels, Pflanzenreich 46(IV.94): 136. 1910. Petala nonnunquam aliquanto maiora, ad $4 \mathrm{~mm}$. longa, $2.5 \mathrm{~mm}$. lata. Mabaduan, Western Division, L. J. Brass 6501, 6477. - Tarara, L. J. Brass 8541.

Known only from New Guinea.

Hypserpa laurina (F. Muell.) Diels, Pflanzenreich 46(IV.94) : 209. 1910.

Sepala intima maiora, 5-nervosa.

Lower Fly River, east bank, opposite Sturt Island, rain forest, large liana of ridges, climbing by tendrillate branches with or without leaves, flowers green, fruits red, Oct. 1936, L. J. Brass 7995. - Tarara, Wasi Kussa River, Western Division, L. J. Brass 8736.

The first record of this species outside N. E. Australia.

Sarcopetalum Harveyanum F. Muell. Pl. Vict. 1:27, 221, suppl. pl. 3. 1860-1862; Diels, Pflanzenreich 46(IV.94): 252. 1910.

Endocarpium 4-6 mm. longum, 5-7 mm. latum ideoque plerumque quam illud formae typicae $(3-3.5 \mathrm{~mm}$. longum, 4-4.5 mm. latum) conspicue amplius, tuberculis maioribus fere aculeolatum.

Lake Daviumbu, Middle Fly River, rain forest, common twiner ascending substage trees, infl. lateral, on lower stem, fr. soft, red, 6-8 mm. diam., Sept. 1936, L. J. Brass 7714. - Tarara, Wassi Kussa River, Western Division, small climber common in rain forests, Jan. 1937, L. J. Brass 8659.

The first record of this monotypic genus outside N. E. Australia. The New Guinean specimens agree completely with those of Australia; only the endocarp is somewhat larger.

\section{ANNONACEAE}

Uvaria micrantha (A. DC.) Hook. f. et Thoms. Fl. Ind. 1: 103. 1855; Hook. f., Fl. Brit. Ind. 1: 51. 1872.

Guatteria micrantha A. DC. Mém. Fam. Anon. 42. 1832.

Tarara, Wassi Kussa River, Western Division, rain forest, liane, fls. purple-brown, Jan. 1937, L. J. Brass 8557. - Kanosia, forest on edge of mangrove swamps, sea level, ca. 8 feet tall, calyx brownish-olive, 
corolla brown, Feb. 2, 1935, C. E. Carr 11088. - Lolorua, forest, ca. 100 feet, climber, fruit orange, Feb. 24, 1935, C. E. Carr 11509.

The species is distributed from Further India and Sumatra to the Philippines. First records for New Guinea.

Rauwenhoffia Leichhardtii (F. Muell.) Diels, comb. nov.

Unona Leichhardtii F. Muell. Fragm. 3: 41. 1842.

Melodorum Leichhardtii Benth. Fl. Austr. 1: 52. 186.3; Bailey, Queensl. Flora 1:25. 1899.

Rauwenhoffia uvarioides Scheffer, Ann. Jard. Buitenz. 2: 23. 1885 ; Diels, Bot. Jahrb. 44: 125. 1912.

Uvaria lutescens K. Schum. in K. Schum. \& Hollr., F1. Kaiser-Wilhelmsl. 48. 1889; K. Schum. \& Lauterb., F1. Deutsch. Schutzgeb. Süds. 315. 1901 .

Lake Daviumbu, Middle Fly River, large climber, common in rain forest canopy, Aug. 1936, L. J. Brass 7584; same locality, flowers yellow-brown, sweet-scented, Sept. 1936, L. J. Brass 7681. - Lower Fly River, east bank opposite Sturt Island, rain forest, common high climbing liane on ridges, fruit orange-brown, Oct. 1936, L. J. Brass 7993.

Mitrephora Versteegii Diels, Bot. Jahrb. 44: 153. 1912.

Southeast New Guinea: Palmer River, two miles below junction Black River, common in rain forest substage $100 \mathrm{~m}$. alt. slender tree attaining 8-9 m., numerous golden-yellow flowers in lateral fascicles on old wood, July 1936, L. J. Brass 7311.

Orophea rhytidocarpa Diels, n. sp.

Arbor ex coll. parva circ. $3 \mathrm{~m}$ alta. Rami juniores pilosi. Foliorum petiolus crassus brevis $0.3-0.4 \mathrm{~cm}$. longus, pilosus, lamina papyracea, glabra, lineari-lanceolata, apicem versus sensim longe angustata, apice ipsa obtusiuscula, basi subobliqua, $30-35 \mathrm{~cm}$. longa, $6-6.5 \mathrm{~cm}$. lata, nervi laterales primarii $12-15$ a costa abeuntes, supra inconspicui, subtus prominuli. Pedunculi axillares, longissimi, penduli. Carpidia pauca (2 ?), breviter (circ. $3 \mathrm{~mm}$.) stipitata, divaricata, subglobosa, dense velutinella, viridia, costulis irregulariter reticulatis conspicue rugosa, viva ex coll. $2.5 \mathrm{~cm}$., sicca circ. $2 \mathrm{~cm}$. diam.

Fly River: 528 mile Camp, forest undergrowth, on ridges, alt. $80 \mathrm{~m}$., small sparsely branched near-tree $3 \mathrm{~m}$. high, leaves secund, a solitary 2-lobed rugose green fruit on a long axillary peduncle (lobes of fruit $2.5 \mathrm{~cm}$. diam.) May 1936, L. J. Brass 6736.

Near O. dolichonema Diels from N. E. New Guinea, but differs in the narrower leaves, the more distant nerves, hardly connected at the margin, and the ripe, strongly corrugated carpels.

Botanical Museum,

Berlin-Dahlem, Germany. 


\section{$2 \mathrm{BHL}$ Biodiversity Heritage Library}

Diels, Ludwig. 1939. "Botanical Results of the Archbold Expeditions New Guinea Records of Annonaceae and Menispermaceae." Journal of the Arnold Arboretum 20(1), 73-74. https://doi.org/10.5962/p.185396.

View This Item Online: $\underline{\text { https://www.biodiversitylibrary.org/item/33596 }}$

DOI: https://doi.org/10.5962/p.185396

Permalink: https://www.biodiversitylibrary.org/partpdf/185396

\section{Holding Institution}

Missouri Botanical Garden, Peter H. Raven Library

\section{Sponsored by}

Missouri Botanical Garden

\section{Copyright \& Reuse}

Copyright Status: In copyright. Digitized with the permission of the rights holder.

Rights Holder: Arnold Arboretum of Harvard University

License: http://creativecommons.org/licenses/by-nc-sa/3.0/

Rights: https://biodiversitylibrary.org/permissions

This document was created from content at the Biodiversity Heritage Library, the world's largest open access digital library for biodiversity literature and archives. Visit BHL at https://www.biodiversitylibrary.org. 